\title{
An approach for solving singular two-point boundary value problems: analytical and numerical treatment
}

\section{Changbum Chun ${ }^{1}$ \\ Abdelhalim Ebaid ${ }^{2}$ Emad Aly ${ }^{4}$ \\ Mi Young Lee ${ }^{3}$}

(Received 11 August 2011; revised 10 March 2012)

\begin{abstract}
The numerical treatment of two-point singular boundary value problems has always been a difficult and challenging task due to the singularity behaviour that occurs at a point. Various efficient numerical methods have been proposed to deal with such boundary value problems. We present a new efficient modification of the Adomian decomposition method for solving singular boundary value problems, both linear and nonlinear. Numerical examples illustrate the efficiency and accuracy of the proposed method.
\end{abstract}

http://journal.austms.org.au/ojs/index.php/ANZIAMJ/article/view/4582 gives this article, (c) Austral. Mathematical Soc. 2012. Published March 20, 2012. ISSN 1446-8735. (Print two pages per sheet of paper.) Copies of this article must not be made otherwise available on the internet; instead link directly to this URL for this article. 


\section{Contents}

1 Introduction

E22

2 A new approach

E24

2.1 Case $\mathrm{I}: \mu(\mathrm{c})=0 \ldots \ldots \ldots \ldots \ldots \ldots$ E25 . . . . . . . . . . .

2.2 Case II: $\mu($ c $)$ otherwise defined $\ldots \ldots \ldots \ldots$ E26 . . . . . .

3 Numerical examples

E28

3.1 Example: inhomogeneous Bessel equation . . . . . . . . E29

3.2 Example: linear singular equation . . . . . . . . . . E E31

3.3 Example: nonlinear singular two-point BVP . . . . . . . E34

3.4 Example: Thomas-Fermi equation . . . . . . . . . E E35

4 Important remark

E36

5 Example of an irregular singularity

E37

6 Conclusions

E39

References

E40

\section{Introduction}

The Adomian decomposition method (ADM), which accurately computes a series solution, is one of the powerful and reliable methods for solving various kinds of linear and nonlinear problems arising in applied sciences $[1,2]$. This method provides the solution in a rapidly convergent series solution and has been successfully applied to a wide class of boundary value problems $[2$, $3,4,5,6,7,8,9,10]$. The convergence of the decomposition series has been investigated by several researchers $[11,12]$. In this work, we obtain 
approximate solutions of the singular two-point boundary value problems [13]

$$
u^{\prime \prime}(x)+p(x) u^{\prime}(x)+q(x) f(u(x))=r(x), \quad x \in(a, b),
$$

subject to the boundary conditions

$$
\mathfrak{u}(\mathrm{a})=\alpha \quad \text { and } \quad \mathfrak{u}(\mathrm{b})=\beta,
$$

where at least one of the functions $p(x), q(x)$ and $r(x)$ has a singular point and $a, b, \alpha$ and $\beta$ are finite constants. Equation (1) is used to model several phenomena in important physics and engineering applications, and contains many well-known models as special cases. When $p(x)=0$ and $f(u(x))=$ $[u(x)]^{-\sigma}$, Equation (1) is known as the generalized Emden-Fowler equation with negative exponent and arises frequently in applied mathematics [14]. In addition, when $p(x)=r(x)=0, q(x)=-x^{-1 / 2}$ and $f(u(x))=[u(x)]^{3 / 2}$, Equation (1) becomes Thomas-Fermi equation [3] which arises in the study of the electrical potential in the atom. Further, with $p(x)=p / x, q(x)=1$ and $r(x)=0$, Equation (1) plays an important role in the analysis of heat conduction through a solid with heat generation [15].

The numerical treatment of singular boundary value problems (BVPs) has always been a difficult and challenging task due to the singular behaviour that occurs at a point. Various efficient numerical methods have been proposed to solve this type of problem $[10,13,16]$. These methods include the homotopy analysis method [17], differential transformation method [16], modified ADM $[18,19,20,21]$, and an improved ADM [13]. The first two of these methods obtained analytical and numerical solutions for some linear singular two-point BVPs, the others successfully solved linear and nonlinear singular two-point BVPs. Often the ADM may require additional calculations in order to determine an unknown constant in any of its $n$-term approximations to the solution [19] and the accuracy of the method decreases. Accordingly, it is desirable to have a modification of the decomposition method which explicitly determines the zeroth component of the solution. One such method was suggested by Ebaid [13], which is based on the ADM and a modification of Lesnic's work [4] to create a canonical form of recursive relation with an 
explicitly determined zeroth component. The efficiency and reliability of this method has been well demonstrated in various singular problems. This motivates the development of another approach which is presented in this paper. In this work a new modification of the Adomian decomposition method is introduced for solving the singular two-point boundary value problem (1)(2). We propose a new inverse operator of the differential operator which easily overcomes the singularity difficulty of the boundary value problem. The present work focuses on creating a canonical form of recursive relation which contains all the boundary conditions so that the zeroth component is explicitly determined without additional computations, as are all other components. The method proposed in this contribution may be considered as a further extension of the method by Ebaid [13] and provides a more accurate approximation than previous methods $[8,13,22]$. Several numerical examples illustrate the accuracy of the proposed method, and the numerical results obtained by it and other efficient methods are presented and compared.

\section{A new approach}

We rewrite Equation (1) in the form

$$
L(u)=r(x)-q(x) f(u(x)),
$$

where the linear differential operator $L$ is defined by

$$
L[.]=\mu(x)^{-1} \frac{d}{d x}\left(\mu(x) \frac{d}{d x}[.]\right), \quad \mu(x)=\exp \left[\int p(x) d x\right] .
$$

To solve the two-point boundary value problem we propose the inverse operator $\mathrm{L}^{-1}$ in the form

$$
L^{-1}[.]=\int_{a}^{x} \mu(t)^{-1} \int_{c}^{t} \mu(z)[.] d z d t+\xi(x) \int_{a}^{b} \mu(t)^{-1} \int_{c}^{t} \mu(z)[.] d z d t
$$


where the constant $\mathrm{c}$ and the function $\xi(x)$ are unknowns and to be determined such that $\mathrm{L}^{-1} \mathrm{~L}(\mathrm{u})$ is expressed in terms of the given boundary conditions. Let us discuss this point. On using the proposed inverse operator defined in (5) we obtain

$$
L^{-1} L(u)=u(x)-\eta(x),
$$

where

$$
\begin{aligned}
\eta(x)= & u(a)-\xi(x)[u(b)-u(a)] \\
& +\mu(c) u^{\prime}(c)\left[\int_{a}^{x} \mu(t)^{-1} d t+\xi(x) \int_{a}^{b} \mu(t)^{-1} d t\right] .
\end{aligned}
$$

Here, two cases for $\eta(x)$ are observed from Equation (7) which depend on the choice of $c$ and $\xi(x)$.

\section{$2.1 \quad$ Case I: $\mu(\mathrm{c})=0$}

If $c$ is a root of the equation $\mu(x)=0$, that is, $\mu(c)=0$, it then follows that

$$
\eta(x)=u(a)-\xi(x)[u(b)-u(a)] .
$$

The function $\xi(x)$ that appears in (8) is chosen to be any function which makes $\eta(x)$ satisfy the given boundary conditions; that is, $\xi(x)$ satisfies the conditions

$$
\xi(a)=0, \quad \xi(b)=-1 .
$$

With this choice of $\xi(x), \eta(x)$ in (8) is expressed in terms of the boundary conditions only, and contain no unknown constants. We have therefore some freedom in choosing the function $\xi(x)$ for defining new different inverse operators and $\eta(x)$ for constructing the different zeroth components of the solution in the recursive ADM relation. This point will be discussed in detail through examples. 


\subsection{Case II: $\mu(\mathrm{c})$ otherwise defined}

If $\mu(x)$ has no zeros, $c$ is chosen so that $\mu(c)$ is defined. In this case we eliminate the term with $\mathfrak{u}^{\prime}(\mathbf{c})$ in order to express $\eta(x)$ in (7) in terms of the boundary conditions only by setting

$$
\int_{a}^{x} \mu(t)^{-1} d t+\xi(x) \int_{a}^{b} \mu(t)^{-1} d t=0 .
$$

Solving this equation for $\xi(x)$, we obtain

$$
\xi(x)=-\frac{\int_{a}^{x} \mu(t)^{-1} d t}{\int_{a}^{b} \mu(t)^{-1} d t} .
$$

Therefore, $\eta(x)$ in Equations (6) and (7) becomes

$$
\eta(x)=u(a)+\frac{\int_{a}^{x} \mu(t)^{-1} d t}{\int_{a}^{b} \mu(t)^{-1} d t}[u(b)-u(a)] .
$$

This $\eta(x)$ is expressed in terms of the boundary conditions only, and contains no unknown constants. The current approach overcomes the singularity difficulty commonly encountered with singular boundary value problems.

With $\eta(x)$ as described in (8) or (12), upon operating $\mathrm{L}^{-1}$ on Equation (3), it then follows that

$$
u(x)=\eta(x)+L^{-1}[r(x)]-L^{-1}[q(x) f(u(x))] .
$$

The Adomian decomposition method introduces the solution $u(x)$ and the nonlinear function $f(u)$ by the infinite series

$$
u(x)=\sum_{n=0}^{\infty} u_{n}(x)
$$

and

$$
f(u)=\sum_{n=0}^{\infty} A_{n}\left(u_{0}, u_{1}, \ldots, u_{n}\right)
$$


where $A_{n}$ are Adomian polynomials for the nonlinear term $f(u(x))$. These polynomials are obtained from

$$
A_{n}=\frac{1}{n !}\left[\frac{d^{n}}{d \lambda^{n}} f\left(\sum_{i=0}^{n} \lambda^{i} u_{i}\right)\right]_{\lambda=0}, \quad n \geqslant 0,
$$

and the first few are

$$
\begin{aligned}
& A_{0}=f\left(u_{0}\right), \\
& A_{1}=u_{1} f^{\prime}\left(u_{0}\right), \\
& A_{2}=u_{2} f^{\prime}\left(u_{0}\right)+\frac{1}{2 !} u_{1}^{2} f^{\prime \prime}\left(u_{0}\right), \\
& A_{3}=u_{3} f^{\prime}\left(u_{0}\right)+u_{1} u_{2} f^{\prime \prime}\left(u_{0}\right)+\frac{1}{3 !} u_{1}^{3} f^{\prime \prime \prime}\left(u_{0}\right), \\
& A_{4}=u_{4} f^{\prime}\left(u_{0}\right)+\left(\frac{1}{2 !} u_{2}^{2}+u_{1} u_{3}\right) f^{\prime \prime}\left(u_{0}\right)+\frac{1}{2 !} u_{1}^{2} u_{2} f^{\prime \prime \prime}\left(u_{0}\right)+\frac{1}{4 !} u_{1}^{4} f^{(4)}\left(u_{0}\right) .
\end{aligned}
$$

The components $u_{n}(x)$ of the solution $u(x)$ will be determined recurrently, and Adomian polynomials can be constructed for various classes of nonlinearity according to specific algorithms presented by Wazwaz [9]. Substituting (14) and (15) into (13) yields

$$
\sum_{n=0}^{\infty} u_{n}(x)=\eta(x)+L^{-1}[r(x)]-L^{-1}\left[q(x) \sum_{n=0}^{\infty} A_{n}\right] .
$$

According to the standard Adomian decomposition method, we have the recurrence relation

$$
\begin{aligned}
& u_{0}(x)=\eta(x)+L^{-1}[r(x)], \\
& u_{n+1}(x)=-L^{-1}\left[q(x) A_{n}(x)\right], \quad n \geqslant 0 .
\end{aligned}
$$


The function $\eta(x)$ is decomposed into two parts, namely $f_{0}(x)$ and $f_{1}(x)$ such that $\eta(x)=f_{0}(x)+f_{1}(x)$. Now we have the following recursive relation for the modified decomposition method [21]

$$
\begin{aligned}
& u_{0}(x)=f_{0}(x), \\
& u_{1}(x)=f_{1}(x)+L^{-1}[r(x)]-L^{-1}\left[q(x) A_{0}(x)\right], \\
& u_{n+1}(x)=-L^{-1}\left[q(x) A_{n}(x)\right], \quad n \geqslant 1,
\end{aligned}
$$

which is used for the determination of the components $\mathfrak{u}_{n}(x)$ of $\mathfrak{u}(x)$. When $r(x)=0$, we obtain the similar but simpler recurrence relation,

$$
\begin{aligned}
& u_{0}(x)=f_{0}(x), \\
& u_{1}(x)=f_{1}(x)-L^{-1}\left[q(x) A_{0}(x)\right], \\
& u_{n+1}(x)=-L^{-1}\left[q(x) A_{n}(x)\right], \quad n \geqslant 1 .
\end{aligned}
$$

Having determined the components $u_{n}(x), n \geqslant 0$, recurrently, the series solution of $\mathfrak{u}(x)$ defined by (14) follows immediately. For numerical purposes, the $n$-term approximation to the exact solution $u(x)$ is

$$
\phi_{\mathfrak{n}}(x)=\sum_{k=0}^{n-1} \mathfrak{u}_{k}(x) .
$$

Based on the remarks made by Wazwaz [21], the success of this modification depends only on the choice of $f_{0}$ and $f_{1}$, and this can be made through trials. Moreover, by proper selection of the function $f_{0}$ and $f_{1}$, the exact solution $u$ is obtained from very few iterations.

\section{$3 \quad$ Numerical examples}

In this section, we consider both linear and nonlinear problems to demonstrate the efficiency and accuracy of the modified algorithm proposed in this contribution. Our computation was performed using the Maple package. 


\subsection{Example: inhomogeneous Bessel equation}

Consider the inhomogeneous Bessel equation [22]

$$
u^{\prime \prime}(x)+\frac{1}{x} u^{\prime}(x)+u(x)=4-9 x+x^{2}-x^{3}, \quad x \in(0,1),
$$

with boundary conditions

$$
\mathfrak{u}(0)=0 \quad \text { and } \quad \mathfrak{u}(1)=0 .
$$

Observe from Equation (23) that $p(x)=1 / x$ and this gives $\mu(x)=x$. From Case I discussed in Section 2.1, $c$ is chosen such that $\mu(\mathrm{c})=0$; that is, $c=0$. $\xi(x)$ is also chosen freely in a way such that the conditions (9) are satisfied; that is, $\xi(0)=0$, and $\xi(1)=-1$. One such choice is $\xi(x)=-x$. There are many other functions for such a purpose; for example, the functions of polynomials type $\xi(x)=-x^{s}$ or $-\chi^{s}\left(x^{\gamma}+1\right) / 2$ such that $s>0, \gamma \geqslant-s$, and functions of trigonometric type $\sin (3 \pi x / 2),-\sin (\pi x / 2)$ and $-\tan (\pi x / 4)$. It is difficult to discuss all possible choices of $\xi(x)$; however, the case $\xi(x)=-\chi^{s}$ is chosen to illustrate the method of solution. In addition, the effect of $s$ on the absolute errors will also be discussed. Firstly, note from Equation (8) that $\eta(x)=0$, consequently the solution obtained from the modified decomposition method is evaluated using the following algorithm

$$
\begin{aligned}
& u_{0}(x)=0, \\
& u_{1}(x)=L^{-1}\left[4-9 x+x^{2}-x^{3}\right]-L^{-1}\left[u_{0}(x)\right], \\
& u_{n+1}(x)=-L^{-1}\left[u_{n}(x)\right], \quad n \geqslant 1,
\end{aligned}
$$

where

$$
\mathrm{L}^{-1}[.]=\int_{0}^{\mathrm{x}} \mathrm{t}^{-1} \int_{0}^{\mathrm{t}} z[.] \mathrm{d} z \mathrm{dt}-\mathrm{x}^{\mathrm{s}} \int_{0}^{1} \mathrm{t}^{-1} \int_{0}^{\mathrm{t}} z[.] \mathrm{d} z \mathrm{dt} .
$$

From this recurrence relation, different approximate analytic solutions $\phi_{\mathfrak{n}}(\chi, s)$ are derived from different values assigned to $s$. In terms of $s$, we calculated 
the four-term approximate solution as

$$
\begin{aligned}
\phi_{4}(x, s)= & u_{0}(x)+u_{1}(x)+u_{2}(x)+u_{3}(x) \\
= & x^{2}-x^{3}+\frac{x^{8}}{36864}-\frac{x^{9}}{99225}-\frac{9 x^{s+4}}{400\left(s^{2}+6 s+8\right)^{2}} \\
& +\left[\frac{13280-9256 s-649 s^{2}}{705600(s+2)^{4}}\right] x^{s+2} \\
& +\left[-\frac{6929}{406425600}-\frac{9}{400(s+2)^{4}}+\frac{649}{705600(s+2)^{2}}\right. \\
& \left.+\frac{9}{400\left(s^{2}+6 s+8\right)^{2}}\right] x^{s} .
\end{aligned}
$$

In this four term approximate solution, we note that as $s$ increases, the last five terms become very small and therefore can be neglected, where $0<x<1$ implies $x^{8}, x^{9}, x^{s}, x^{s+2}, x^{s+4} \ll 1$. This shows that as $s$ increases finitely or infinitely $\phi_{4}(x, s)$ becomes close to $\left(x^{2}-x^{3}\right)$, which is the exact solution. Note that as $s \rightarrow \infty$ we have the following limits:

$$
\begin{aligned}
\lim _{s \rightarrow \infty} & {\left[\frac{9 x^{s+4}}{400\left(s^{2}+6 s+8\right)^{2}}\right]=0, } \\
\lim _{s \rightarrow \infty} & {\left[\frac{13280-9256 s-649 s^{2}}{705600(s+2)^{4}}\right] x^{s+2}=0, } \\
\lim _{s \rightarrow \infty} & {\left[-\frac{6929}{406425600}-\frac{9}{400(s+2)^{4}}\right.} \\
& \left.+\frac{649}{705600(s+2)^{2}}+\frac{9}{400\left(s^{2}+6 s+8\right)^{2}}\right] x^{s}=0 .
\end{aligned}
$$

Therefore,

$$
\begin{aligned}
\lim _{s \rightarrow \infty} \phi_{4}(x, s) & =x^{2}-x^{3}+2.71267 \times 10^{-5} \chi^{8}-1.00781 \times 10^{-5} \chi^{9} \\
& \approx x^{2}-x^{3}, \quad \text { for all } x \in(0,1) .
\end{aligned}
$$


Moreover, it is expected that the accuracy of the approximate solution increases as the number of terms in the Adomian series increases. The ten term approximate analytic solutions $\phi_{10}(x, 1)$ and $\phi_{10}(x, 3)$ are

$$
\begin{aligned}
\phi_{10}(x, 1)= & \sum_{k=0}^{9} \mathfrak{u}_{k}(x), \\
= & -2.2 \times 10^{-12} x+x^{2}-x^{3}-3.5 \times 10^{-12} x^{5}+1.3 \times 10^{-12} x^{7} \\
& -3.1 \times 10^{-13} x^{9}+4.7 \times 10^{-14} x^{11}-5.1 \times 10^{-15} x^{13} \\
& +3.8 \times 10^{-16} x^{15}-1.9 \times 10^{-17} x^{17}+2.9 \times 10^{-19} x^{20} \\
& -4.8 \times 10^{-20} x^{21}
\end{aligned}
$$

and

$$
\begin{aligned}
\phi_{10}(x, 3)= & x^{2}-\chi^{3}+2.6 \times 10^{-17} \chi^{5}-5.3 \times 10^{-17} \chi^{7}+1.2 \times 10^{-17} \chi^{11} \\
& -1.5 \times 10^{-17} \chi^{13}+4.6 \times 10^{-18} \chi^{15}-1.5 \times 10^{-19} \chi^{17} \\
& -4.7 \times 10^{-19} \chi^{19}+2.9 \times 10^{-19} \chi^{20}-4.7 \times 10^{-20} \chi^{21}
\end{aligned}
$$

which are also in excellent agreement with the exact solution $u(x)=x^{2}-x^{3}$. Table 1 compares the absolute errors obtained by Ebaid, Cui and Geng [13, 22], and the modified decomposition method proposed in this contribution at $s=1$ and $s=3$, respectively. Importantly, this table shows the accuracy increases with increasing $s$, especially at $s=3$ in which a very small absolute error is obtained, where the error is assumed to be zero for an exponent less than -16 . Hence, $\left|\mathfrak{u}(x)-\phi_{10}(x, 3)\right|$ is considered as zero in Table 1. This clearly shows the improvements and the accuracy we achieved compared to the methods discussed by Ebaid, Cui and Geng [13, 22].

\subsection{Example: linear singular equation}

Consider the linear singular equation [22]

$$
x^{2} u^{\prime \prime}(x)-x u^{\prime}(x)+u(x)=0, \quad 1 \leqslant x \leqslant 2,
$$


Table 1: Absolute errors for Example 3.1, where $\left|\mathfrak{u}(\mathbf{x})-\mathfrak{u}_{26}\right|$ was discussed by Cui and Geng [22] and $\left|\mathfrak{u}(x)-\Phi_{10}(x)\right|$ was discussed by Ebaid [13].

\begin{tabular}{ccccc}
\hline$x$ & $\left|\mathfrak{u}(x)-\mathfrak{u}_{26}\right|$ & $\left|\mathfrak{u}(x)-\Phi_{10}(x)\right|$ & $\left|\mathfrak{u}(x)-\phi_{10}(x, 1)\right|$ & $\left|\mathfrak{u}(x)-\phi_{10}(x, 3)\right|$ \\
\hline 0.0 & 0 & 0 & 0 & 0 \\
0.1 & $2.3 \times 10^{-5}$ & $2.2 \times 10^{-5}$ & $2.2 \times 10^{-13}$ & 0 \\
0.2 & $1.1 \times 10^{-5}$ & $2.5 \times 10^{-5}$ & $4.1 \times 10^{-13}$ & 0 \\
0.3 & $5.5 \times 10^{-5}$ & $3.1 \times 10^{-5}$ & $5.6 \times 10^{-13}$ & 0 \\
0.4 & $2.3 \times 10^{-4}$ & $2.4 \times 10^{-5}$ & $6.3 \times 10^{-13}$ & 0 \\
0.5 & $1.1 \times 10^{-4}$ & $1.7 \times 10^{-5}$ & $6.4 \times 10^{-13}$ & 0 \\
0.6 & $1.2 \times 10^{-4}$ & $1.1 \times 10^{-5}$ & $5.8 \times 10^{-13}$ & 0 \\
0.7 & $1.6 \times 10^{-4}$ & $7.0 \times 10^{-6}$ & $4.6 \times 10^{-13}$ & 0 \\
0.8 & $1.5 \times 10^{-4}$ & $4.0 \times 10^{-6}$ & $3.1 \times 10^{-13}$ & 0 \\
0.9 & $4.1 \times 10^{-5}$ & $1.8 \times 10^{-6}$ & $1.5 \times 10^{-13}$ & 0 \\
1.0 & 0 & 0 & $3.8 \times 10^{-19}$ & 0
\end{tabular}

with boundary conditions

$$
u(1)=1 \quad \text { and } \quad \mathfrak{u}(2)=1 .
$$

The exact solution is

$$
u(x)=x-\frac{x \ln (x)}{2 \ln (2)}, \quad 1 \leqslant x \leqslant 2 .
$$

In this example, we have $p(x)=-1 / x$, and this gives $\mu(x)=x^{-1}$ which has no zeros. The present example follows Case II (§2.2). Accordingly, we choose $c$ such that $\mu(\mathrm{c})$ is defined, that is, $c \in \mathbb{R}-\{0\}$. Equations (11) and (12) can now be used to compute $\xi(x)$ and $\eta(x)$ as

$$
\xi(x)=-\frac{\int_{1}^{x} \mu(t)^{-1} d t}{\int_{1}^{2} \mu(t)^{-1} d t}=\frac{1}{3}\left(1-x^{2}\right),
$$

and

$$
\eta(x)=u(1)+\frac{\int_{1}^{x} \mu(t)^{-1} d t}{\int_{1}^{2} \mu(t)^{-1} d t}[u(2)-u(1)]=1 .
$$


Table 2: Absolute errors for Example 3.2.

\begin{tabular}{cccc}
\hline$\chi$ & $\left|\mathrm{u}-\mathrm{S}_{7}\right|[8]$ & $\left|\mathrm{u}-\Phi_{7}\right|[13]$ & $\left|\mathrm{u}(\mathrm{x})-\phi_{7}\right|$ \\
\hline 1.0 & 0 & 0 & 0 \\
1.1 & $2.6 \times 10^{-8}$ & $2.6 \times 10^{-8}$ & $2.0 \times 10^{-10}$ \\
1.2 & $5.4 \times 10^{-8}$ & $5.4 \times 10^{-8}$ & $3.9 \times 10^{-10}$ \\
1.3 & $6.8 \times 10^{-8}$ & $6.8 \times 10^{-8}$ & $5.4 \times 10^{-10}$ \\
1.4 & $6.4 \times 10^{-8}$ & $6.4 \times 10^{-8}$ & $6.2 \times 10^{-10}$ \\
1.5 & $4.5 \times 10^{-8}$ & $4.5 \times 10^{-8}$ & $6.4 \times 10^{-10}$ \\
1.6 & $2.0 \times 10^{-8}$ & $2.0 \times 10^{-8}$ & $6.0 \times 10^{-10}$ \\
1.7 & $1.8 \times 10^{-9}$ & $1.8 \times 10^{-8}$ & $5.1 \times 10^{-10}$ \\
1.8 & $1.4 \times 10^{-8}$ & $1.4 \times 10^{-8}$ & $3.7 \times 10^{-10}$ \\
1.9 & $1.4 \times 10^{-8}$ & $1.4 \times 10^{-8}$ & $1.9 \times 10^{-10}$ \\
2.0 & 0 & 0 & 0 \\
\hline & & &
\end{tabular}

The decomposition method given in (19) admits the use of the recursive relation

$$
\begin{aligned}
& u_{0}(x)=1, \\
& u_{n+1}(x)=-L^{-1}\left[\frac{1}{x^{2}} u_{n}(x)\right], \quad n \geqslant 0,
\end{aligned}
$$

where

$$
\mathrm{L}^{-1}[.]=\int_{1}^{x} \mathrm{t} \int_{c}^{t} z^{-1}[.] \mathrm{d} z \mathrm{dt}+\frac{1}{3}\left(1-\chi^{2}\right) \int_{1}^{2} \mathrm{t} \int_{c}^{t} z^{-1}[.] \mathrm{d} z \mathrm{dt}, \quad c \in \mathbb{R}-\{0\} .
$$

Table 2 compares the absolute errors obtained using the modified ADM with $\mathrm{c}=1$ proposed in this contribution, the extended ADM [8] and the improved ADM [13]. For the considered example, the present method is much more accurate than these effective methods $[8,13]$. 


\subsection{Example: nonlinear singular two-point BVP}

Consider the nonlinear singular two-point boundary value problem [23, 24]

$$
u^{\prime \prime}(x)+\frac{0.5}{x} u^{\prime}(x)=e^{\mathfrak{u}(x)}\left(0.5-e^{\mathfrak{u}(x)}\right), \quad x \in(0,1),
$$

subject to the boundary conditions

$$
\mathfrak{u}(0)=\ln 2 \text { and } \mathfrak{u}(1)=0 .
$$

The exact solution is

$$
u(x)=\ln \left(\frac{2}{x^{2}+1}\right) .
$$

Proceeding as above, we obtain $\mu(x)=x^{1 / 2}$, hence $c=0$. According to Case I $(\S 2.1), \xi(x)$ is an arbitrary function and to be chosen such that $\xi(0)=0$ and $\xi(1)=-1$. As discussed in Example 3.1, the function $\xi(x)$ can be chosen as $\xi(x)=-\chi^{s}, s>0$, and the effect of $s$ on the absolute errors will be discussed later. From Equation (8), $\eta(x)=\left(1-x^{s}\right) \ln 2$, consequently the solution obtained from the modified decomposition method is evaluated using

$$
\begin{aligned}
& u_{0}(x)=\ln 2, \\
& u_{1}(x)=-\ln 2 x^{s}+L^{-1}\left[A_{0}\right], \\
& u_{n+1}(x)=L^{-1}\left[A_{n}\right], \quad n \geqslant 1,
\end{aligned}
$$

where

$$
\mathrm{L}^{-1}[.]=\int_{0}^{x} \mathrm{t}^{-1 / 2} \int_{0}^{\mathrm{t}} z^{1 / 2}[.] \mathrm{d} z \mathrm{dt}-x^{s} \int_{0}^{1} \mathrm{t}^{-1 / 2} \int_{0}^{\mathrm{t}} z^{1 / 2}[.] \mathrm{d} z \mathrm{dt} .
$$

The three term approximate solution is calculated in terms of $\mathrm{s}$ as

$$
\begin{aligned}
\phi_{3}(x, s)= & \mathfrak{u}_{0}(x)+\mathfrak{u}_{1}(x)+\mathfrak{u}_{2}(x) \\
= & \ln 2-x^{2}+\frac{x^{4}}{2}+\left[\frac{14(-1+\ln 2)}{2 s^{2}+7 s+6}\right] x^{s+2} \\
& -\left[\frac{-34+40 \ln 2+7 s(-1+2 \ln 2)+2 s^{2}(-1+2 \ln 2)}{2\left(2 s^{2}+7 s+6\right)}\right] x^{s} .
\end{aligned}
$$


Table 3: Absolute errors for Example 3.3, where $\left|\mathfrak{u}(\mathbf{x})-\Psi_{3}(\mathrm{x})\right|$ was discussed by Kumar and Singh [10] and $\operatorname{err}(\mathrm{s})=\left|\mathfrak{u}(x)-\phi_{3}(x, s)\right|$

\begin{tabular}{ccccc}
\hline$x$ & $\left|\mathfrak{u}(x)-\Psi_{3}(x)\right|$ & $\operatorname{err}(1)$ & $\operatorname{err}(3)$ & $\operatorname{err}(5)$ \\
\hline 0.0 & 0 & 0 & 0 & 0 \\
0.1 & $3.3 \times 10^{-7}$ & $9.0 \times 10^{-3}$ & $9.8 \times 10^{-5}$ & $1.3 \times 10^{-6}$ \\
0.2 & $2.1 \times 10^{-5}$ & $1.6 \times 10^{-2}$ & $7.9 \times 10^{-4}$ & $2.6 \times 10^{-5}$ \\
0.3 & $2.3 \times 10^{-4}$ & $2.0 \times 10^{-2}$ & $2.6 \times 10^{-3}$ & $1.4 \times 10^{-4}$ \\
0.4 & $1.2 \times 10^{-3}$ & $2.0 \times 10^{-2}$ & $6.0 \times 10^{-3}$ & $3.5 \times 10^{-4}$ \\
0.5 & $4.4 \times 10^{-3}$ & $1.5 \times 10^{-2}$ & $1.0 \times 10^{-2}$ & $5.4 \times 10^{-4}$ \\
0.6 & $1.2 \times 10^{-2}$ & $6.4 \times 10^{-3}$ & $1.6 \times 10^{-2}$ & $3.9 \times 10^{-4}$ \\
0.7 & $2.9 \times 10^{-2}$ & $4.1 \times 10^{-3}$ & $2.1 \times 10^{-2}$ & $4.1 \times 10^{-4}$ \\
0.8 & $5.9 \times 10^{-2}$ & $1.3 \times 10^{-2}$ & $2.2 \times 10^{-2}$ & $1.8 \times 10^{-3}$ \\
0.9 & $1.1 \times 10^{-1}$ & $1.3 \times 10^{-2}$ & $1.6 \times 10^{-2}$ & $2.6 \times 10^{-3}$ \\
1.0 & $1.9 \times 10^{-1}$ & 0 & 0 & 0 \\
\hline
\end{tabular}

Observe from this series solution that the last two terms tend to 0 as $s$ increases. Moreover, the first three terms are exactly those of Taylor series of the exact solution. We also conclude that the accuracy of the numerical solution increases as $s$ increases as shown in Table 3 using only three terms. The accuracy of the numerical solution can be enhanced by increasing the number of terms in the decomposition series solution.

\subsection{Example: Thomas-Fermi equation}

Here we consider the Thomas-Fermi equation

$$
u^{\prime \prime}(x)=x^{-1 / 2} u^{3 / 2}(x), \quad x \in(0,1),
$$

with the boundary conditions

$$
u(0)=1, \quad u(1)=0 .
$$


Note that no exact solution is known for this singular BVP. In order to solve Equations (45) and (46), we first note that $\mu(x)=1$, which has no zeros. Choose $\mathrm{c}$ so that $\mu(\mathrm{c})$ is defined. According to this suggestion, we have

$$
\xi(x)=-\frac{\int_{0}^{x} d t}{\int_{0}^{1} d t}=-x,
$$

and

$$
\eta(x)=u(0)+\frac{\int_{0}^{x} d t}{\int_{0}^{1} d t}[u(1)-u(0)]=1-x .
$$

The modified decomposition method admits the use of the recursive relation

$$
\begin{aligned}
& u_{0}=1, \\
& u_{1}=-x+L^{-1}\left[x^{-1 / 2} A_{0}(x)\right], \\
& u_{n+1}=L^{-1}\left[x^{-1 / 2} A_{n}(x)\right], \quad n \geqslant 1,
\end{aligned}
$$

where

$$
\mathrm{L}^{-1}[.]=\int_{0}^{x} \int_{c}^{t}[.] \mathrm{d} z \mathrm{dt}-x \int_{0}^{1} \int_{c}^{t}[.] \mathrm{d} z \mathrm{dt}, \quad c \in \mathbb{R},
$$

and the $A_{n}$ are Adomian polynomials for the nonlinear function $f(u)=u^{3 / 2}$. The scheme obtained above is exactly the same as Ebaid's [13], where rapid convergence of the resulting approximate solutions was obtained as the number of terms in the decomposition series solution increased. Thus our method in this article may be considered an extension of the method presented by Ebaid [13].

\section{Important remark}

In the previous section, examples with singular points of regular type are discussed and the present approach is found effective for handling such kinds 
of singular two-point BVPs. However, difficulties arise when applying the proposed approach to the general Bessel differential equation $x^{2} u^{\prime \prime}(x)+x u^{\prime}(x)+$ $\left(x^{2}-n^{2}\right) u(x)=0, n \neq 0$, when the singular point $(x=0)$ is an end point or within the interval of interest, noting that $x=0$ remains a regular singular point. For the purpose of illustration, assume that $\mathfrak{u}(0)=\alpha$ and $\mathfrak{u}(1)=\beta$ are two boundary conditions for the general Bessel differential equation. On applying the current approach as shown in the previous examples, the modified decomposition method admits the use of the recursive relation

$$
\begin{aligned}
& u_{0}(x)=\alpha, \\
& u_{1}(x)=(\alpha-\beta) \xi(x)-L^{-1}\left[\left(1-\frac{n^{2}}{x^{2}}\right) u_{0}(x)\right], \\
& u_{n+1}(x)=-L^{-1}\left[\left(1-\frac{n^{2}}{x^{2}}\right) u_{n}(x)\right], \quad n \geqslant 1,
\end{aligned}
$$

where

$$
\mathrm{L}^{-1}[.]=\int_{0}^{x} \mathrm{t}^{-1} \int_{0}^{\mathrm{t}} z[.] \mathrm{d} z \mathrm{dt}+\xi(\mathrm{x}) \int_{0}^{1} \mathrm{t}^{-1} \int_{0}^{\mathrm{t}} z[.] \mathrm{d} z \mathrm{dt},
$$

and $\xi(x)$ is left open and can be specified as illustrated in Example 3.1. Observe from Equation (51) that the term $L^{-1}\left[\left(n^{2} / x^{2}\right) u_{0}(x)\right]$ cannot be evaluated using the inverse operator defined in (52), where the first fold integration does not exist, and therefore further modification is needed in this case. Such modification is based on changing the independent variable $x$ as $x=1-y$. Details are discussed below through an example in which $p(x)=-1 / x^{2}$, where the singularity at $x=0$ is of irregular type.

\section{Example of an irregular singularity}

Consider the two-point boundary value problem

$$
u^{\prime \prime}(x)-\frac{1}{x^{2}} u^{\prime}(x)=\frac{1}{x^{2}}, \quad u(0)=1, \quad u(1)=0
$$


for which $p(x)=-1 / x^{2}$. We now show the difficulty of applying the standard procedure discussed in the previous examples. As previously explained, we should first obtain $\mu(x)$. Note that $\mu(x)=e^{1 / x}$, hence

$$
\xi(x)=-\frac{\int_{0}^{x} \mu(t)^{-1} d t}{\int_{0}^{1} \mu(t)^{-1} d t}=\frac{\Gamma(0,-1 / x)-x e^{-1 / x}}{1 / e-\operatorname{Ei}(-1)},
$$

where $\Gamma(\mathrm{a}, z)=\int_{z}^{\infty} \mathrm{t}^{\mathrm{a}-1} e^{-\mathrm{t}} \mathrm{dt}$ and $\operatorname{Ei}(z)=-\int_{-z}^{\infty} e^{-\mathrm{t}} / \mathrm{t} d \mathrm{t}$ are the incomplete Gamma function and exponential integral function, respectively. Therefore it is difficult to proceed with the standard procedure due to difficulties arising from evaluating these integrals.

Instead, we propose a new independent variable $y$ as $x=1-y$ and accordingly the present BVP becomes

$$
\begin{aligned}
& u^{\prime \prime}(y)+\frac{1}{(1-y)^{2}} u^{\prime}(y)=\frac{1}{(1-y)^{2}}, \\
& u(0)=0, \quad u(1)=1
\end{aligned}
$$

Now it is possible to remove the singularity by using the series substitution $(1-y)^{-2}=\sum_{n=0}^{\infty}(n+1) y^{n}$ in Equation (55) to give

$$
u^{\prime \prime}(y)+\left(\sum_{n=0}^{\infty}(n+1) y^{n}\right) u^{\prime}(y)=\sum_{n=0}^{\infty}(n+1) y^{n} .
$$

On applying the improved ADM, as suggested Ebaid [13], to Equation (57) and using the boundary conditions in (56), we obtain the recursive relation

$$
\begin{aligned}
& u_{0}(y)=y, \\
& u_{n+1}(y)=L^{-1}\left[(n+1) y^{n}-\sum_{k=0}^{n}(k+1) y^{k} u_{n-k}^{\prime}(y)\right], \quad n \geqslant 0,
\end{aligned}
$$

where

$$
\mathrm{L}^{-1}[.]=\int_{0}^{y} \int_{c}^{t}[.] \mathrm{d} z \mathrm{dt}-\mathrm{y} \int_{0}^{1} \int_{c}^{t}[.] \mathrm{d} z \mathrm{dt}, \quad c \in \mathbb{R} .
$$


At $n=0$,

$$
u_{1}(y)=L^{-1}\left(1-u_{0}^{\prime}(y)\right)=0,
$$

and at $\mathrm{n}=1$ we obtain

$$
u_{2}(y)=L^{-1}\left[2 y-u_{1}^{\prime}-2 y u_{0}^{\prime}\right]=0 .
$$

Proceeding as above we obtain

$$
u_{n}(y)=0, \quad \text { for all } n \geqslant 1 .
$$

Therefore, the solution is given only by the zeroth component,

$$
\mathrm{u}(\mathrm{y})=\mathrm{y} .
$$

In terms of the original independent variable $x$, we obtain

$$
u(x)=1-x,
$$

which is the exact solution of the current irregular singular two-point BVP.

\section{Conclusions}

We presented an efficient modified Adomian decomposition method to solve linear and nonlinear two-point boundary value problems with a singular feature. One of the main characteristics of the present approach is that all the boundary conditions of the problem are included in the recursive relation and the difficulty of singular problems, due to the existence of a singular point, is easily overcome. It was demonstrated that the proposed approach can be well suited to attaining an accurate solution to singular two-point boundary value problems, both linear and nonlinear. Other types of differential equations with singular features can also be similarly handled by the proposed method. 
Acknowledgements This research was supported by the Basic Science Research Program through the National Research Foundation of Korea funded by the Ministry of Education, Science and Technology (2011-0025877).

\section{References}

[1] G. Adomian. A review of the decomposition method and some recent results for nonlinear equation. Math. Comput. Modelling, 3, 1992, 17-43. $\mathrm{E} 22$

[2] G. Adomian. Solving frontier problems of physics: the decomposition method. Kluwer Academic Publishers, Boston, 1994. E22

[3] G. Adomian. Solution of the Thomas-Fermi equation. Appl. Math. Lett., 11(3), 1998, 131-133. E22, E23

[4] D. Lesnic. A computational algebraic investigation of the decomposition method for time-dependent problems. Appl. Math. Comput., 119, 2001, 197-206. E22, E23

[5] E. Babolian and J. Biazar. Solving the problem of biological species living together by Adomian decomposition method. Appl. Math. Comput., 129, 2002, 339-343. E22

[6] M. Benabidallah and Y. Cherruault. Application of the Adomian method for solving a class of boundary problems. Kybernetes, 33, 2004, 118-132. E22

[7] E. H. Aly, A. Ebaid and R. Rach. Advances in the Adomian decomposition method for solving two-point nonlinear boundary value problems with Neumann boundary conditions. Compu. Math. Applic., 63, 2012, 1056-1065. E22 
[8] B. Jang. Two-point boundary value problems by the extended Adomian decomposition method. J. Comput. Appl. Math., 219, 2008, 253-263. E22, E24, E33

[9] A. M. Wazwaz. Partial differential equations and solitary waves theory. Springer, New York, 2009. E22, E27

[10] M. Kumar and N. Singh. Modified Adomian decomposition method and computer implementation for solving singular boundary value problems arising in various physical problems. Comput. Chem. Eng., 34, 2010, 1750-1760. E22, E23, E35

[11] Y. Cherruault, G. Adomian, K. Abbaoui and R. Rach. Further remarks on convergence of decomposition method. Bio-Medical Comput., 38, 1995, 89-93. E22

[12] M. M. Hosseini and H. Nasabzadeh. On the convergence of Adomian decomposition method. Appl. Math. Comput., 182, 2006, 536-543. E22

[13] A. Ebaid. A new analytical and numerical treatment for singular two-point boundary value problems via the Adomian decomposition method. J. Comput. Appl. Math., 235, 2011, 1914-1924. E23, E24, E31, $\mathrm{E} 32, \mathrm{E} 33, \mathrm{E} 36, \mathrm{E} 38$

[14] J. Janus and J. Myjak. A generalized Emden-Fowler equation with a negative exponent. Nonlin. Analy., 23, 1994, 953-970. E23

[15] M. K. Kadalbajoo and V. K. Aggarwal. Numerical solution of singular boundary value problems via Chebyshev polynomial and B-spline. Appl. Math. Comput., 160, 2005, 851-863. E23

[16] A. S. V. Ravi Kanth and K. Aruna. Solution of singular two-point boundary value problems using differential transformation method. Phys. Lett. A, 372, 2008, 4671-4673. E23 
[17] Sami Bataineh, M. S. M. Noorani and I. Hashim. Approximate solutions of singular two-point BVPs by modified homotopy analysis method. Phys. Lett. A, 372, 2008, 4062-4066. E23

[18] A. M. Wazwaz. Adomian decomposition method for a reliable treatment of the Emden-Fowler equation. Appl. Math. Comput., 161, 2005, 543-560. E23

[19] M. Inc, M. Ergut and Y. Cherruault. A different approach for solving singular two-point boundary value problems. Kybernetes, 34, 2005, 934-940. E23

[20] C. Chun. A modified Adomian decomposition method for solving higher-order singular boundary value problems. Z. Naturforsch. A, 65, 2010, 1093-1100. E23

[21] A. M. Wazwaz. The modified decomposition method for analytic treatment of differential equations. Appl. Math. Comput., 173, 2006, 165-176. E23, E28

[22] M. Cui and F. Geng. Solving singular two-point boundary value problem in reproducing kernel space. J. Comput. Appl. Math., 205, 2007, 6-15. E24, E29, E31, E32

[23] S. M. El-Sayed. Integral methods for computing solutions of a class of singular two-point boundary value problems. Appl. Math. Comput., 130, 2002, 235-241. E34

[24] A. Ebaid. Exact solutions for a class of nonlinear singular two-point boundary value problems: The decomposition method.

Z. Naturforsch. A, 65, 2010, 145-150. E34 


\section{Author addresses}

1. Changbum Chun, Department of Mathematics, Sungkyunkwan University, Suwon 440-746, RePublic of KoreA mailto: cbchun@skku.edu

2. Abdelhalim Ebaid, Department of Mathematics, Faculty of Science, Tabuk University, P.O. Box 741, Tabuk 71491, SAUdi ArABIA mailto:halimgamil@yahoo.com

3. Mi Young Lee, Department of Mathematics, Sungkyunkwan University, Suwon 440-746, Republic of KoreA mailto: cbchun@skku .edu

4. Emad Aly, Department of Mathematics, Faculty of Science, King Abdulaziz University, Jeddah, SAUdi Arabia; Permanent address: Department of Mathematics, Faculty of Education, Ain Shams University, Roxy, Cairo, Egypt. mailto: emad-aly@hotmail.com 